\title{
Toward optical sensing of metal nanoparticles using chiral sculptured thin films
}

\author{
Akhlesh Lakhtakia \\ Department of Engineering Science and Mechanics, Pennsylvania State University, University \\ Park, PA 16802-6812, USA \\ akhlesh@psu.edu
}

\begin{abstract}
A nominal model wherein the nanowires of a chiral sculptured thin film (STF) are considered as strings of slender ellipsoids indicates that the circular Bragg phenomenon evinced by chiral STFs can be exploited to optically sense small concentrations of metal nanoparticles.
\end{abstract}

Keywords: circular Bragg phenomenon, gold, metal, nanocolloid, nanoparticle, sculptured thin film, silver

\section{INTRODUCTION}

Inorganic clusters of linear dimensions between 1 to $3 \mathrm{~nm}$ coalesce to form ensembles of parallel, shaped nanowires during physical vapor deposition (PVD) on a suitably mobile substrate at appropriate temperature and pressure, the ensembles being called sculptured thin films (STFs) [1]. Many optical applications of STFs as polarization filters [2,3] and sensors [4,5] have been established within the past few years, and improved techniques to deposit STFs continue to emerge [6-8].

The nanowires of a chiral STF are helical in shape, which is responsible for the display of circular Bragg phenomenon that is exploited for circular-polarization-rejection filters $[1,2]$ as well as circularly-polarized-light emitters [9]. STFs are porous materials. Infiltration of the void regions of a chiral STF by a fluid leads to a shift of the circular Bragg regime $[4,10,11]$, depending on the porosity of the thin film and the dielectric contrast between the nanohelixes and the infiltrant fluid.

This theoretical communication is premised on the systematic alteration of the optical characteristics of chiral STFs after infiltration by metal nanoparticles, and provides a theoretical argument for the sensing of metal nanoparticles by exploiting the spectral shifts of the circular Bragg phenomenon. Metals provide an uncommon dielectric contrast to the inorganic materials that many STFs today are made of. The relative permittivities of oxides and fluorides and other commonly used organic and inorganic optical materials have positive real parts in the visible regime [12]. The relative permittivities of fluids generally have similar characteristics. In contrast, the relative permittivities of metals have negative real parts in the same wavelengthregime [13].

The optical response characteristics of a chiral STF infiltrated uniformly with metal particles can be theoretically predicted by (i) first using a local-homogenization model to obtain the reference relative permittivity dyadic [1, Ch. 6], and (ii) then exploiting the standard solution of a matrix ordinary differential equation to solve a boundary-value problem [1, Ch. 9]. The localhomogenization model is based on the celebrated Bruggeman formalism. This formalism yields physically implausible results when the volumetric fraction of the metal is significantly high in a two-constituent composite material $[14,15]$. However, that implausibility is not a significant issue in the present context, because the volume fraction of the metal is not expected to exceed 0.01 . The nanoparticles are supposed to be spheres of diameters around $20 \mathrm{~nm}$, so that they are electrically small in the visible regime but are not so small that a relative permittivity cannot be prescribed for them [16]. 
The plan of this communication is as follows. Section 2 contains brief descriptions of the relative permittivity dyadic of a chiral STF infiltrated uniformly with the chosen nanoparticles, the local-homogenization model for a three-constituent composite medium, and the boundaryvalue problem for the reflection and transmission of obliquely incident plane waves by a metalinfiltrated chiral STF of finite thickness. Numerical results are presented and discussed in Sec. 3 . Vectors are in boldface, dyadics are double-underlined. An $\exp (-i \omega t)$ time-dependence is implicit with $i=\sqrt{-1}, \omega$ as the angular frequency, and $t$ as time.

\section{THEORY IN BRIEF}

Suppose the region $0 \leq z \leq L$ is occupied by a chiral STF that is uniformly inflitrated by spherical nanoparticles. The permittivity dyadic of infiltrated thin film may be stated as [1, Ch. 9]

$$
\underline{\underline{\epsilon}}(\mathbf{r})=\epsilon_{0} \underline{\underline{S}}_{z}(h, z, \Omega) \cdot \underline{\underline{S}}_{y}(\chi) \cdot \underline{\epsilon}_{r e f}^{o} \cdot \underline{\underline{S}}_{y}^{-1}(\chi) \cdot \underline{\underline{S}}_{z}^{-1}(h, z, \Omega), \quad 0 \leq z \leq L .
$$

In this equation, $\epsilon_{0}=8.854 \times 10^{-12} \mathrm{~F} \mathrm{~m}^{-1}$ is the free-space permittivity; $\underline{\epsilon}_{r e f}^{o}=\epsilon_{a} \mathbf{u}_{z} \mathbf{u}_{z}+$ $\epsilon_{b} \mathbf{u}_{x} \mathbf{u}_{x}+\epsilon_{c} \mathbf{u}_{y} \mathbf{u}_{y}$ is the reference relative permittivity dyadic, with $\mathbf{u}_{x}, \mathbf{u}_{y}$ and $\mathbf{u}_{z}$ are cartesian unit vectors; the tilt dyadic $\underline{S}_{y}(\chi)=\mathbf{u}_{y} \mathbf{u}_{y}+\left(\mathbf{u}_{x} \mathbf{u}_{x}+\mathbf{u}_{z} \mathbf{u}_{z}\right) \cos \chi+\left(\mathbf{u}_{z} \mathbf{u}_{x}-\mathbf{u}_{x} \mathbf{u}_{z}\right) \sin \chi$ represents the locally aciculate microstructure with the angle $\chi \in(0, \pi / 2]$; the rotation dyadic

$$
\underline{\underline{S}}_{z}(h, z, \Omega)=\mathbf{u}_{z} \mathbf{u}_{z}+\left(\mathbf{u}_{x} \mathbf{u}_{x}+\mathbf{u}_{y} \mathbf{u}_{y}\right) \cos \left(h \frac{\pi z}{\Omega}\right)+\left(\mathbf{u}_{y} \mathbf{u}_{x}-\mathbf{u}_{x} \mathbf{u}_{y}\right) \sin \left(h \frac{\pi z}{\Omega}\right)
$$

involves $2 \Omega$ as the structural period; and the parameter $h=1$ for structural right-handedness and $h=-1$ for structural left-handedness. The ratio $L / \Omega$ is generally taken to be an integer for theoretical research.

The three scalars $\epsilon_{a, b, c}$ have to be predicted by a microscopic-to-continuum approach. The application of a local-homogenization model for STFs [1, Ch. 6] proceeds as follows. Suppose that a material of relative permittivity $\epsilon_{1}$ is deposited using a PVD process [2] as a chiral STF of thickness $L$, with $f_{1},\left(0<f_{1}<1\right)$, being the volume fraction occupied by the deposited material. The parameters $\Omega, \chi$ and $h$ are fixed during the deposition process along with $f_{1}$. Next, let the film be infiltrated uniformly by nanoparticles of a metal of relative permittivity $\epsilon_{2}$, the corresponding volume fraction being denoted by $f_{2},\left(0<f_{2}<1\right)$. The void regions of the infiltrated thin film are vacuous and their volume fraction is denoted by $f_{3}=1-f_{2}-f_{3}$ such that $0<f_{3}<1$. Thus, the metal-infiltrated chiral STF is a three-constituent composite material [17], in contrast to a fluid-infiltrated chiral STF which can be treated as a two-constituent composite material $[4,18]$.

In the local-homogenization model, the deposited material is supposed to be present in the form of electrically small ellipsoids with a transverse aspect ratio $\gamma_{t}$ somewhat larger than unity and a slenderness ratio $\gamma_{s}>>1$; i.e., every nanowire is a string of ellipsoidal sausages. The infiltrant metal is assumed to be present in the form of electrically small spheres, and the void regions are also thought of as electrically small spheres. The infiltrated film is divided into thin sections in which all the ellipsoids are identically oriented, and the Bruggeman equation

$$
f_{1} \underline{\underline{a}}_{1}+f_{2} \underline{\underline{a}}_{2}+f_{3} \underline{\underline{a}}_{3}=\underline{\underline{0}}
$$

is solved. Here,

$$
\underline{\underline{a}}_{j}=\epsilon_{0}\left(\epsilon_{j} \underline{\underline{I}}-\underline{\underline{\epsilon}}_{r e f}^{o}\right) \cdot\left[\underline{\underline{I}}+i \omega \epsilon_{0} \underline{\underline{D}}_{j} \cdot\left(\epsilon_{j} \underline{\underline{I}}-\underline{\underline{\epsilon}}_{r e f}^{o}\right)\right]^{-1}, \quad j=1,2,3,
$$


are polarizability density dyadics; the depolarization dyadics are given by [10]

$$
\begin{aligned}
\underline{\underline{D}}_{1}= & \frac{1}{i \omega \epsilon_{0}} \frac{2}{\pi} \int_{\varphi=0}^{\pi / 2} \int_{\vartheta=0}^{\pi / 2} \sin \vartheta \\
& \times\left[\frac{\left.\frac{\frac{\cos ^{2} \vartheta}{\gamma_{s}^{2}} \mathbf{u}_{x} \mathbf{u}_{x}+\sin ^{2} \vartheta\left(\cos ^{2} \varphi \mathbf{u}_{z} \mathbf{u}_{z}+\frac{\sin ^{2} \varphi}{\gamma_{t}^{2}} \mathbf{u}_{y} \mathbf{u}_{y}\right)}{\epsilon_{b} \frac{\cos ^{2} \vartheta}{\gamma_{s}^{2}}+\sin ^{2} \vartheta\left(\epsilon_{a} \cos ^{2} \varphi+\epsilon_{c} \frac{\sin ^{2} \varphi}{\gamma_{t}^{2}}\right)}\right] d \vartheta d \varphi,}{},\right.
\end{aligned}
$$

and

$$
\underline{\underline{D}}_{2}=\underline{\underline{D}}_{3}=\left.\underline{\underline{D}}_{1}\right|_{\gamma_{s}=\gamma_{t}=1}
$$

$\underline{\underline{0}}$ is the null dyadic; $\underline{\underline{I}}$ is the identity dyadic; and $\epsilon_{3}=1$. Equation (3) has to be solved numerically for $\underline{\epsilon}_{r e f}^{o}$.

The plane wave incident on the chiral STF is delineated by the electric field phasor [1]

$$
\mathbf{E}_{i n c}(z)=\left(a_{L} \frac{i \mathbf{s}-\mathbf{p}_{+}}{\sqrt{2}}-a_{R} \frac{i \mathbf{s}+\mathbf{p}_{+}}{\sqrt{2}}\right) e^{i k_{0} z \cos \theta} e^{i k_{0}(x \cos \phi+y \sin \phi) \sin \theta}, \quad z \leq 0,
$$

where $a_{L}$ and $a_{R}$ are the known amplitudes of the left- and the right-circularly polarized (LCP and RCP), respectively; the vectors $\mathbf{s}=-\mathbf{u}_{x} \sin \phi+\mathbf{u}_{y} \cos \phi$ and $\mathbf{p}_{ \pm}=\mp\left(\mathbf{u}_{x} \cos \phi+\mathbf{u}_{y} \sin \phi\right)$ $\cos \theta+\mathbf{u}_{z} \sin \theta$ are of unit magnitude; the propagation vector of the incident plane wave makes an angle $\theta \in[0, \pi / 2)$ with respect to the $+z$ axis, and is inclined to the $x$ axis in the $x y$ plane by an angle $\psi \in[0,2 \pi)$; and $k_{0}=\omega \sqrt{\epsilon_{0} \mu_{0}}$ is the free-space wavenumber with $\mu_{0}=4 \pi \times 10^{-7} \mathrm{H} \mathrm{m}^{-1}$ as the free-space permeability. The free-space wavelength is denoted by $\lambda_{0}=2 \pi / k_{0}$. The electric field phasors associated with the reflected and transmitted plane waves, respectively, are expressed by [1]

$$
\mathbf{E}_{r e f}(z)=\left(-r_{L} \frac{i \mathbf{s}-\mathbf{p}_{-}}{\sqrt{2}}+r_{R} \frac{i \mathbf{s}+\mathbf{p}_{-}}{\sqrt{2}}\right) e^{-i k_{0} z \cos \theta} e^{i k_{0}(x \cos \phi+y \sin \phi) \sin \theta}, \quad z \leq 0,
$$

and

$$
\mathbf{E}_{t r}(z)=\left(t_{L} \frac{i \mathbf{s}-\mathbf{p}_{+}}{\sqrt{2}}-t_{R} \frac{i \mathbf{s}+\mathbf{p}_{+}}{\sqrt{2}}\right) e^{i k_{0}(z-L) \cos \theta} e^{i k_{0}(x \cos \phi+y \sin \phi) \sin \theta} \cdot \quad z \geq L .
$$

The amplitudes $r_{L, R}$ and $t_{L, R}$ indicate the as-yet unknown strengths of the LCP and RCP components of the reflected and transmitted plane waves.

Numerical techniques to determine $r_{L}, r_{R}, t_{L}$ and $t_{R}$ in terms of $a_{L}$ and $a_{R}$ have been described elsewhere in detail $[1,19,20]$. Those techniques involve the solution of a $4 \times 4$ matrix ordinary differential equation and yield the reflection and transmission coefficients that appear as the elements of the $2 \times 2$ matrixes in the following relations:

$$
\left[\begin{array}{c}
r_{L} \\
r_{R}
\end{array}\right]=\left[\begin{array}{ll}
r_{L L} & r_{L R} \\
r_{R L} & r_{R R}
\end{array}\right]\left[\begin{array}{c}
a_{L} \\
a_{R}
\end{array}\right], \quad\left[\begin{array}{c}
t_{L} \\
t_{R}
\end{array}\right]=\left[\begin{array}{cc}
t_{L L} & t_{L R} \\
t_{R L} & t_{R R}
\end{array}\right]\left[\begin{array}{c}
a_{L} \\
a_{R}
\end{array}\right] .
$$

Co-polarized coefficients have both subscripts identical, but cross-polarized coefficients do not. The square of the magnitude of a reflection or transmission coefficient is the corresponding reflectance or transmittance; thus, $R_{L R}=\left|r_{L R}\right|^{2}$ is the reflectance corresponding to the reflection coefficient $r_{L R}$, and so on. The principle of conservation of energy mandates the constraints $R_{L L}+R_{R L}+T_{L L}+T_{R L} \leq 1$ and $R_{R R}+R_{L R}+T_{R R}+T_{L R} \leq 1$, the inequalities turning to equalities only in the absence of dissipation in the region $0<z<L$.

The outlined approach to predict the reflectances and transmittances requires calibration against experimental results. Whereas $h, \Omega$ and $L$ are set during PVD, and $\chi$ can be ascertained 


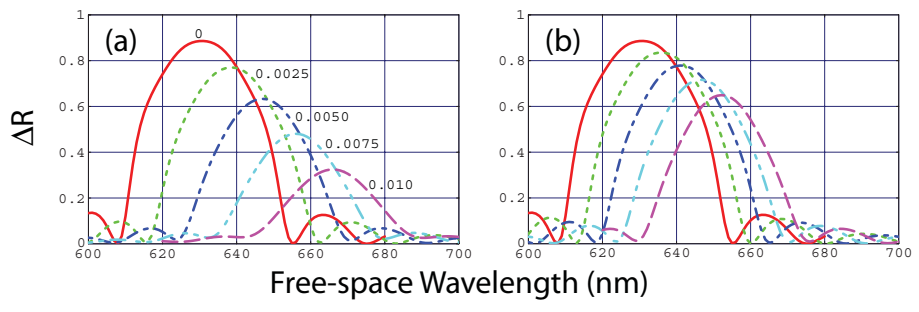

Fig. 1. $\Delta R$ versus $\lambda_{0}$ for $\epsilon_{1}=5, f_{1}=0.5, \Omega=200 \mathrm{~nm}, L=32 \Omega, \chi=30^{\circ}, \phi=$ $0^{\circ}$, and $\theta=10^{\circ}$. The volume fraction of the infiltrated metal is 0 (solid), 0.0025 (dotted), 0.005 (dash-dotted), 0.0075 (dash-dot-dotted), or 0.01 (dashed). (a) $\epsilon_{2}=-9.98+0.85 i$, (b) $\epsilon_{2}=-17.4+2.25 i$. Similar results were obtained with $\epsilon_{2}=-3.12+19.2 i$ (iron at $\left.\lambda_{0}=610 \mathrm{~nm}[13]\right)$.

using scanning electron microscopy, spectrums of the reflectances and transmittances of some uninfiltrated chiral STFs need to be used to choose values of $\gamma_{s}$ and $\gamma_{t}$ [18]; furthermore, $\epsilon_{1}$ may have to be adjusted somewhat from the known value of the bulk relative permittivity of the nanohelix material [18]. Next, comparison against the spectrums of the reflectances and transmittances of some metal-infiltrated chiral STFs may be necessary to adjust $\epsilon_{2}$ from the known value of the thin-film relative permittivity [13] of the metal. These steps are not expected to significantly affect qualitative conclusions that can be drawn from preliminary theoretical studies.

\section{NUMERICAL RESULTS AND DISCUSSION}

Figure 1 shows computed values of the quantity $\Delta R=h\left(R_{R R}-R_{L L}+R_{L R}-R_{R L}\right)$ versus the free-space wavelength $\lambda_{0}$, when $\epsilon_{1}=5$ (typical of titanium oxide in the visible regime [12]), $f_{1}=0.5, \Omega=200 \mathrm{~nm}, L=32 \Omega, \chi=30^{\circ}, \phi=0^{\circ}$, and $\theta=10^{\circ}$. The volume fraction $f_{2}$ of the metal varies from 0 to 0.01 , and either $\epsilon_{2}=-9.98+0.85 i$ (gold at $\lambda_{0}=610 \mathrm{~nm}$ [13]) or $\epsilon_{2}=-17.4+2.25 i$ (silver at $\lambda_{0}=610 \mathrm{~nm}$ [13]).

The large humps of $\Delta R$ centered at $\lambda_{0} \approx 631 \mathrm{~nm}$ for $f_{2}=0$ in both Figs. 1(a) and (b) indicate the circular Bragg phenomenon in the uninfiltrated chiral STF. The humps narrow and tend towards a top-hat profile as $L / \Omega$ increases further. Also, the humps are more pronounced for smaller values of $\theta$ than for larger values of $\theta$; indeed, $\Delta R$ is known to vanish as $\theta$ increases above, say, $45^{\circ}[21]$.

As the volume fraction $f_{2}$ of the metal increases, the circular Bragg regime in Fig. 1 executes a redshift. Simultaneously, the peak value of $\Delta R$ in the circular Bragg regime also decreases. These two findings suggest that chiral STFs can be used to sense the concentration of metal particles in colloids and suspensions [22]. Furthermore, the redshift of the $\Delta R$-peak is more pronounced in Fig. 1(a) than in Fig. 1(b): on the average about $4000 \mathrm{~nm}$ per unit volume fraction (UVF) for gold nanoparticles, and about $2000 \mathrm{~nm} \mathrm{UVF}^{-1}$ for silver nanoparticles. Additionally, the diminishment of the $\Delta R$-peak is enhanced in Fig. 1(a) in relation to Fig. 1(b). These observations suggest that chiral STFs may also be useful in sensing the type of infiltrant metal.

A somewhat different device to sense the type and concentration of metal nanoparticles emerges if the top half of the chiral STF were to be rotated about the $z$ axis by $90^{\circ}$ with respect to the bottom half $[4,23]$. The introduction thereby of the central $90^{\circ}$-twist defect creates a sharp dip in the spectrum of $\Delta R$ right in the center of the circular Bragg regime [24]. The calculated spectrums of $\Delta R$ for this device are shown in Fig. 2.

Whereas variations in $\phi$ do not matter significantly [1], a value of $\theta$ somewhat different from $0^{\circ}$ may be preferable for designing a chiral-STF-based spectroscopic device to sense metal 


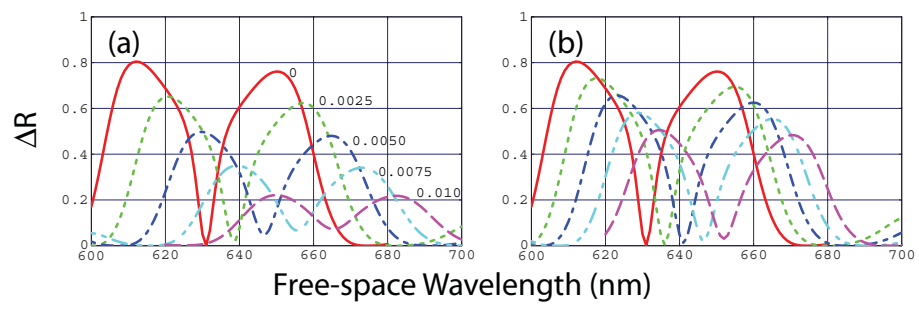

Fig. 2. Same as Fig. 1, except that the top half of the chiral STF is rotated about the $z$ axis by $90^{\circ}$ with respect to the bottom half.

nanoparticles by measuring $\Delta R$. If dissipation inside the metal-infiltrated chiral STF remains low enough, spectrums of $\Delta T=h\left(T_{L L}-T_{R R}+T_{R L}-T_{L R}\right)$ may also be similarly useful.

The experimentally recorded spectrums of $\Delta R$ are expected to be somewhat different from the theoretical ones presented in Figs. 1 and 2 for three reasons: First, the relative permittivity of the infiltrating metal (as also of the nanohelix material) is frequency-dependent. If necessary, that feature can be incorporated in the local-homogenization model. Second, some fluid may also have to be inserted along with the metal nanoparticles in the void regions of chiral STFs. That feature too can be taken into account in the local-homogenization model. Third, the metal nanoparticles have been assumed to infiltrate the chiral STF uniformly, which may not be completely realistic. However, the volume fraction of the metal is going to be low enough that a somewhat nonuniform distribution may not have significant consequences.

Metal nanoparticles are being used for a variety of applications such as catalysis, nanoplasmonics, and biosensing [25]. Suspensions and colloids of metal nanoparticles would need to be made for infiltrating STFs [16]. This communication is expected to be of use for the metrology of nanoparticles. It also provides a route for post-deposition engineering of the optical responses of STFs.

\section{References}

[1] A. Lakhtakia and R. Messier, Sculptured Thin Films: Nanoengineered Morphology and Optics, SPIE Press, Bellingham, WA, USA (2005).

[2] Q. Wu, I. J. Hodgkinson, and A. Lakhtakia, "Circular polarization filters made of chiral sculptured thin films: experimental and simulation results," Opt. Eng. 39, 1863-1868 (2000) [doi:10.1117/1.602570].

[3] I. J. Hodgkinson, Q. H. Wu, K. E. Thorn, et al., "Spacerless circular-polarization spectralhole filters using chiral sculptured thin films: theory and experiment," Opt. Commun. 184, 57-66 (2000) [doi:10.1016/S0030-4018(00)00935-4].

[4] A. Lakhtakia, M. W. McCall, J. A. Sherwin, et al., "Sculptured-thin-film spectral holes for optical sensing of fluids," Opt. Commun. 194, 33-46 (2001) [doi:10.1016/S00304018(01)01225-1].

[5] H. Tan, O. Ezekoye, J. van der Schalie, et al., "Biological reduction of nanoengineered iron(III) oxide sculptured thin films," Environ. Sci. Technol. 40, 5490-5495 (2006) [doi:10.1021/es060388j S0013-936X(06)00388-9].

[6] S. Pursel, M. W. Horn, M. C. Demirel, and A. Lakhtakia, "Growth of sculptured polymer submicronwire assemblies by vapor deposition," Polymer 46, 9544-9548 (2005) [doi:10.1016/j.polymer.2005.07.092].

[7] S. V. Kesapragada and D. Gall, "Anisotropic broadening of Cu nanorods during glancing angle deposition," Appl. Phys. Lett. 89, 203121 (2006) [doi:10.1063/1.2388861].

[8] H. E. Ruda, J. C. Polanyi, J. (S. Y.) Yang, et al., "Developing 1D nanostructure arrays for future nanophotonics," Nanoscale Res. Lett. 1, 99-119 (2006) [doi:10.1007/s11671-0069016-6]. 
[9] J. Xu, A. Lakhtakia, J. Liou, A. Chen, and I. J. Hodgkinson, "Circularly polarized fluorescence from light-emitting microcavities with sculptured-thin-film chiral reflectors," Opt. Commun. 264, 235-239 (2006) [doi:10.1016/j.optcom.2006.02.025].

[10] A. Lakhtakia, "Enhancement of optical activity of chiral sculptured thin films by suitable infiltration of void regions," Optik 112 (2001), 145-148 (2001) [doi:10.1078/0030-402600024]; correction: 112, 544 (2001).

[11] S. R. Kennedy, J. C. Sit, D. J. Broer, and M. J. Brett, "Optical activity of chiral thin film and liquid crystal hybrids," Liq. Cryst. 28, 1799-1803 (2001) [doi: 10.1080/02678290110076399].

[12] H. A. Macleod, Thin-Film Optical Filters, Institute of Physics, Bristol, United Kingdom (2001).

[13] J. H. Weaver, C. Krafka, D. W. Lynch, and E. E. Koch, Physik Daten, Physics Data: Optical Properties of Metals, Vols. 18-1 \& 18-2, Fach-informations-zentrum, Karlsruhe, Germany (1981).

[14] T. G. Mackay and A. Lakhtakia, "A limitation of the Bruggeman formalism for homogenization," Opt. Commun. 234, 35-42 (2004) [doi:10.1016/j.optcom.2004.02.007].

[15] T. G. Mackay, "On the effective permittivity of silver-insulator nanocomposites," $J$. Nanophoton. 1, 019501 (2007) [doi:10.1117/1.2472372].

[16] S. A. Kalele, N. R. Tiwari, S. W. Gosavi, and S. K. Kulkarni, "Plasmon-assisted photonics at the nanoscale," J. Nanophoton. 1, 012501 (2007) [doi:10.1117/1.2748429].

[17] T. G. Mackay and A. Lakhtakia, "Percolation thresholds in the homogenization of spheroidal particles oriented in two directions," Opt. Commun. 259, 727-737 (2006) [doi:10.1016/j.optcom.2005.09.070].

[18] J. A. Sherwin, A. Lakhtakia, and I. J. Hodgkinson, "On calibration of a nominal structureproperty relationship model for chiral sculptured thin films by axial transmittance measurements," Opt. Commun. 209, 369-375 (2002) [doi:10.1016/S0030-4018(02)01672-3].

[19] M. Schubert and C. M. Herzinger, "Ellipsometry on anisotropic materials: Bragg conditions and phonons in dielectric helical thin films," Phys. stat. sol. (a) 188, 1563-1575 (2001) [doi:10.1002/1521-396X(200112)188:4<1563::AID-PSSA1563>3.0.CO;2-8].

[20] J. A. Polo, Jr. and A. Lakhtakia, "Comparison of two methods for oblique propagation in helicoidal bianisotropic mediums," Opt. Commun. 230, 369-386 (2004) [doi:10.1016/j.optcom.2003.11.024].

[21] M. D. Pickett, A. Lakhtakia, and J. A. Polo, Jr., "Spectral responses of gyrotropic chiral sculptured thin films to obliquely incident plane waves," Optik 115, 393-398 (2004) [doi:10.1087/0030-4026-00387].

[22] E. V. Shevchenko, D. V. Talapin, C. B. Murray, and S. O'Brien, "Structural characterization of self-assembled multifunctional binary nanoparticle superlattices, J. Am. Chem. Soc. 128, 3620-3637 (2006) [doi:10.1021/ja0564261 S0002-7863(05)06426-7].

[23] S. M. Pursel, M. W. Horn, and A. Lakhtakia, "Tuning of sculptured-thin-film spectral-hole filters by postdeposition etching," Opt. Eng. 46, 040507 (2007) [doi:10.1117/1.272154].

[24] F. Wang and A. Lakhtakia, "Specular and nonspecular, thickness-dependent, spectral holes in a slanted chiral sculptured thin film with a central twist defect," Opt. Commun. 215, 7992 (2003) [doi:10.1016/S0030-4018(02)02204-6].

[25] D. L. Feldheim and C. A. Foss, Jr. (Eds.), Metal Nanoparticles: Synthesis, Characterization \& Applications, Marcel Dekker, New York, NY, USA (2002).

Akhlesh Lakhtakia is the Charles Godfrey Binder Professor of Engineering Science and Mechanics at the Pennsylvania State University. His current research interests include nanotechnology, complex materials, metamaterials, and sculptured thin films. 